be given $\left(r \geqq s ;\left(a_{1}^{\prime}, \cdots, a_{s}^{\prime}\right)\right.$ may be the null set). Then (2.4) and (2.5) associate with (7.1)

$$
\left(p_{1}, \cdots, p_{n}\right) \text { and }\left(p_{1}^{\prime}, \cdots, p_{n}^{\prime}\right)
$$

respectively. Since the primary set (7.1) are distinct, there must be an $a_{i}$ such that $a_{i} \notin\left\{a_{1}^{\prime}, \cdots, a_{s}^{\prime}\right\}$. The $n$-tuplets (7.2) will be distinct since $p_{a_{i}} \neq p_{a_{i}}^{\prime}$. Therefore the lattice points associated with the primary sets (7.1) will be distinct.

City College, New York

\title{
SUBGROUPS OF THE UNIMODULAR GROUP1
}

IRVING REINER

Following the notation of [3], we let $\Gamma$ denote the proper unimodular group consisting of all $2 \times 2$ matrices with rational integral elements and determinant +1 . For $m$ a positive integer, define the principal congruence group $\Gamma(m)$ by

$$
\Gamma(m)=\{X \in \Gamma: X \equiv I(\bmod m)\},
$$

where $I$ denotes the identity matrix in $\Gamma$, and where congruence of matrices is interpreted as elementwise congruence.

For $p$ a prime, we know from [2] that $\Gamma(p)$ is a free group with a finite set $S$ of generators. If we define

$$
T_{m}=\left(\begin{array}{cc}
1 & m \\
0 & 1
\end{array}\right)
$$

then $S$ may be chosen to include $T_{p}$. For each fixed integer $s$, we may define a group $\Omega(p, s)$ consisting of all power products of the generators in $S$ for which the exponent sum for each generator is a multiple of $s$. In [3] it was shown that each $\Omega(p, s)$ is a normal subgroup of $\Gamma$ of finite index in $\Gamma$. Furthermore, if $s>1$ and $(s, p)=1$, it was proved that $\Omega(p, s)$ does not contain any principal congruence group.

Let $\Delta(m)$ denote the normal subgroup of $\Gamma$ which is generated by $T_{m}$. Obviously $\Delta(m) \subset \Gamma(m)$. Recently, Brenner [1] raised the following questions:
A. Does $\Delta(m)=\Gamma(m)$ for all $m$ ?

Received by the editors May 2, 1960.

1 This research was supported by the Office of Naval Research. 
B. For each $m$ does there exist a positive integer $k$ such that $\Delta(m) \supset \Gamma(m k)$ ?

The purpose of this note is to show that the answer to both questions is "No."

Theorem. If $m>1$ and $m$ is not a power of a prime, then $\Delta(m)$ does not contain any principal congruence group.

Proof. From the hypothesis we may write

$$
m=p^{r} s, \quad r \geqq 1, \quad s>1, \quad(p, s)=1 .
$$

Since $T_{m}$ is a power of $T_{p s}$, we have $\Delta(m) \subset \Delta(p s)$. Further, $T_{p s}=T_{p}^{s}$ implies that $T_{p s} \in \Omega(p, s)$. But $\Omega(p, s)$ is a normal subgroup of $\Gamma$, and so we conclude

$$
\Delta(m) \subset \Delta(p s) \subset \Omega(p, s) .
$$

Since $\Omega(p, s)$ contains no principal congruence group, the same holds for $\Delta(m)$. Q.E.D.

Brenner showed that $\Delta(m)=\Gamma(m)$ for $1 \leqq m \leqq 5$. The above theorem implies that $\Delta(6)$ contains no principal congruence group. We are thus left with the following problem: For prime power values of $m$, can $\Delta(m)$ contain a principal congruence group?

The only additional light we can shed on this problem comes from Frasch [2], who showed:

Let $p \geqq 7, p$ prime. Then $\Delta(p)$ is properly contained in $\Gamma(p)$.

\section{REFERENCES}

1. J. L. Brenner, The linear homogeneous group III, Ann. of Math. vol. 71 (1960) pp. 210-223.

2. H. Frasch, Die Erzeugenden der Hauptkongruenzgruppen für Primzahlstufen, Math. Ann. vol. 108 (1933) pp. 229-252.

3. I. Reiner, Normal subgroups of the unimodular group, Illinois J. Math. vol. 2 (1958) pp. 142-144.

UNIVERSITY OF ILLINOIS 